\title{
A Tauberian theorem for Ingham summation method
}

\author{
Vytas Zacharovas \\ Institute of Statistical Science \\ Academia Sinica \\ Taipei 115 \\ Taiwan
}

November 12, 2018

\begin{abstract}
The aim of this work is to prove a Tauberian theorem for the Ingham summability method. The Tauberian theorem we prove is then applied to analyze asymptotics of mean values of multiplicative functions on natural numbers. Key words: Ingham summability, Tauberian theorems, mean values, multiplicative functions.
\end{abstract}

\section{Introduction}

Many problems in number theory involve estimating mean values

$$
\frac{1}{n} \sum_{m=1}^{n} f(m)
$$

of some complex valued function $f: \mathbb{N} \rightarrow \mathbb{C}$. In many cases $f(m)$ can be naturally represented as a sum $\sum_{k \mid m} a_{k}$ where $a_{k} \in \mathbb{C}$. Möbius inversion formula guarantees that for a given $f(m)$ such $a_{k}$ always exist and are unique. Replacing $f(m)$ by $\sum_{k \mid m} a_{k}$ in the sum of values of $f(m)$, we get

$$
\sum_{m=1}^{n} f(m)=\sum_{m=1}^{n} \sum_{k \mid m} a_{k}=\sum_{k=1}^{n} a_{k}\left[\frac{n}{k}\right]
$$


here $[x]$ denotes the integer part of a real number $x$. Suppose we want to know under which conditions the sequence of the mean values (1) of $f(m)$ has a limit as $n \rightarrow \infty$. This is equivalent to the question, under which conditions on $a_{k}$ the sequence

$$
\frac{1}{n} \sum_{k=1}^{n} a_{k}\left[\frac{n}{k}\right]
$$

has a limit as $n \rightarrow \infty$. If, say

$$
\sum_{k \geqslant 1} \frac{\left|a_{k}\right|}{k}<\infty
$$

then the theorem of Wintner (see e. g. [9]) states that

$$
\lim _{n \rightarrow \infty} \frac{1}{n} \sum_{k=1}^{n} a_{k}\left[\frac{n}{k}\right]=\sum_{k=1}^{\infty} \frac{a_{k}}{k} .
$$

It was shown in [4] that the convergence of the series

$$
\sum_{k=1}^{\infty} \frac{a_{k}}{k}
$$

alone, does not necessarily imply the existence of the limit of sum (2) as $n \rightarrow$ $\infty$. In 1910 Axer [1] (see also Chapter 3.6 of [9]) proved that if in addition to convergence of the series (4) the condition

$$
\sum_{k=0}^{n}\left|a_{k}\right|=O(n)
$$

is satisfied, then the limit (3) exists.

We will show that in determining whether the sum (2) has a limit, an important role is played by the quantity

$$
S(x)=\sum_{m \leqslant x} \sum_{k \mid m} a_{k} \log k=\sum_{k \leqslant x} a_{k}\left[\frac{x}{k}\right] \log k .
$$

We will prove (see Lemma 2.3) that condition $S(x)=O(x \log x)$ as $x \rightarrow \infty$ is enough to ensure that the Dirichlet series $\sum_{m=1}^{\infty} a_{m} m^{-\sigma}$ converges for all $\sigma>1$. Which means that the function

$$
g(\sigma)=\sum_{m=1}^{\infty} \frac{a_{m}}{m^{\sigma}}
$$

will be correctly defined in the infinite interval $\sigma>1$. The next theorem shows that if $S(x)=o(n \log n)$ then the value of the sum (2) can be approximated by the values Dirichlet series $g(\sigma)$ with $\sigma=1+\log ^{-1} n$. 
Theorem 1.1. Suppose $a_{n}$ is a sequence of complex numbers such that

$$
S(n)=\sum_{k \leqslant n} a_{k}\left[\frac{n}{k}\right] \log k=o(n \log n) .
$$

Then

$$
\frac{1}{n} \sum_{k \leqslant n} a_{k}\left[\frac{n}{k}\right]=g\left(1+\frac{1}{\log n}\right)+o(1)
$$

as $n \rightarrow \infty$.

The estimate of the above theorem will allow us to prove necessary and sufficient conditions for existence of limit of sum (2).

Theorem 1.2. Suppose $a_{m}$ is a fixed sequence of complex numbers. Then the limit

$$
\lim _{n \rightarrow \infty} \frac{1}{n} \sum_{k \leqslant n} a_{k}\left[\frac{n}{k}\right]=C
$$

exists if and only if the following two conditions are satisfied

1.

$$
\sum_{k \leqslant n} a_{k}\left[\frac{n}{k}\right] \log k=o(n \log n), \quad \text { as } \quad n \rightarrow \infty
$$

2.

$$
\lim _{\sigma \searrow 1} \sum_{m=1}^{\infty} \frac{a_{m}}{m^{\sigma}}=C
$$

Note that if condition $\square$ is satisfied then the infinite series in the formulation of condition 2 converges for all $\sigma>1$.

The last theorem is a direct analog of the very first Tauberian theorem that was proved by Tauber in 1897.

Theorem A (Tauber, [10]). A series

$$
\sum_{n=0}^{\infty} a_{n}
$$

converges and its sum is equal to $A$, if and only if

$$
\sum_{k=0}^{n} k a_{k}=o(n)
$$

and exists the limit

$$
\lim _{x \nearrow 1} \sum_{n=0}^{\infty} a_{n} x^{n}=A .
$$


It can be shown that Tauber's condition (9) imposed on coefficients $a_{j}$ of the formal series (8) alone is enough to provide an asymptotic estimate for partial sums

$$
\sum_{k=0}^{n} a_{k}=\phi\left(e^{-1 / n}\right)+o(1),
$$

where $\phi(z)=\sum_{j=0}^{\infty} a_{j} z^{j}$. Which is similar to the asymptotic given in the formulation of Theorem 1.1 .

It is but natural to ask how really useful are the stated theorems for analyzing the mean values of concrete arithmetical functions? Condition $\lim _{x \downarrow 1} g(x)=C$ does not cause any problem if say the Dirichlet series $g(s)$ has a closed form expression which allows us to obtain information on behavior of $g(x)$ for real values of $x>1$ which are close to 1 . At a first glance the condition $S(n)=$ $o(n \log n)$ looks quite artificial and not much easier to check than to prove that $A(n)=\sum_{k=1}^{n} a_{k}\left[\frac{n}{k}\right]=C n+o(n)$, since $S(n)$ is obtained just by replacing $a_{k}$ by $a_{k} \log k$ in the expression of $A(n)$. However, this condition is quite natural for a wide class of sequences $a_{m}$ such that $f(m)$ defined as $f(m)=\sum_{d \mid m} a_{d}$ is a completely multiplicative function of $m$, that is a function satisfying equation

$$
f(m n)=f(m) f(n),
$$

for any $m, n \in \mathbb{N}$. It is easy to check that if a completely multiplicative function $f$ is bounded $|f(m)| \leqslant 1$, then the condition $S(n)=o(n \log n)$ will be satisfied if

$$
\sum_{p \leqslant n} \frac{|f(p)-1|}{p} \log p=o(\log n),
$$

or

$$
\sum_{m \leqslant n}\left|\sum_{p \leqslant n / m} f(p) \log p-\frac{n}{m}\right|=o(n \log n),
$$

here and further we will follow the tradition to denote by $\sum_{p}$ and $\prod_{p}$ the sums and products over prime numbers $p$. This allows us to deduce a few classical results for the mean values of multiplicative functions. For example, it can be shown that if any of the above two conditions (11) or (12) is satisfied for a fixed multiplicative function $f$ such that $|f(m)| \leqslant 1$, then Theorem 1.1 implies an estimate

$$
\frac{1}{n} \sum_{m=1}^{n} f(m)=\prod_{p} \frac{1-\frac{1}{p^{1+1 / \log n}}}{1-\frac{f(p)}{p^{1+1 / \log n}}}+o(1),
$$

as $n \rightarrow \infty$.

Results with similar or even stronger error terms than in the inequality of the next theorem can be proven by the method of Halász (see e.g. Chapter 19 of 
monograph [2] and papers [3],[7],[8] and [6]). We present its proof bellow just to demonstrate the connection between the Ingham summation method and the mean values of multiplicative functions. Its proof is an easy consequence of the the same estimates that enable us to prove Theorem 1.2 .

Theorem 1.3. Suppose $f(m)$ - completely multiplicative function such that $|f(m)| \leqslant$ 1 then

$$
\left|\frac{1}{n} \sum_{m=1}^{n} f(m)-\prod_{p \leqslant n} \frac{1-\frac{1}{p}}{1-\frac{f(p)}{p}}\right| \leqslant R(\alpha) \mu_{n}(\alpha),
$$

for any $\alpha>1$, with $R(\alpha)$ - a positive constant, which depends on $\alpha$ only, and

$$
\mu_{n}(\alpha)=\left(\frac{1}{\log n} \sum_{p \leqslant n} \frac{|f(p)-1|^{\alpha}}{p} \log p\right)^{1 / \alpha}
$$

Similar result holds for general multiplicative functions, i. e. such functions that condition (10) is required to be satisfied only for coprime pairs of natural numbers $m, n$. It follows from our proof of Theorem 1.3 that its modified version for general multiplicative functions will hold if we weaken condition $|f(m)| \leqslant 1$ to requirement that $|f(1)+f(2)+\cdots f(m)| \leqslant D m$ for all $m \geqslant 1$, with some fixed $D$.

Unfortunately our proof of Theorems 1.1 and 1.2 is not elementary since it relies on the estimate of the number of primes in short intervals (Theorem 2.1) that has originally been proved (see e.g. [5]) using a number of non-trivial facts about distribution of zeroes of the Riemann Zeta function.

The Tauberian theorem we prove can be reformulated in terms of the theory of summation of divergent series. Recall that a formal series $\sum_{m=1}^{\infty} c_{m}$ is called summable in the sense of Ingham if there exists a complex number $C$ such that

$$
\lim _{n \rightarrow \infty} \sum_{m=1}^{n} \frac{m}{n}\left[\frac{n}{m}\right] c_{m}=C,
$$

in which case we write

$$
\text { (I) } \sum_{m=1}^{\infty} c_{m}=C .
$$

Suppose $0<\lambda_{1}<\lambda_{2}<\cdots \lambda_{n}<\cdots$ is a sequence of positive strictly increasing real numbers. Then we say that a formal series $\sum_{m=0}^{\infty} c_{m}$ is $\left(A, \lambda_{n}\right)$ summable and its value is $C$ if

$$
\lim _{x \downarrow 0} \sum_{m=0}^{\infty} c_{m} e^{-\lambda_{m} x}=C,
$$


in which case we write

$$
\left(A, \lambda_{n}\right) \sum_{m=1}^{\infty} c_{m}=C .
$$

With these notations our tauberian theorem means that $(I) \sum_{m=1}^{\infty} c_{m}=C$ if and only if

$$
\sum_{m=1}^{n} \frac{m}{n}\left[\frac{n}{m}\right] c_{m} \log m=o(\log n), \quad \text { as } \quad n \rightarrow \infty
$$

and $(A, \log n) \sum_{m=1}^{\infty} c_{m}=C$.

The analogy between the classical Tauber's theorem and the theorem we prove leads us to expect that a wide class of summability methods is connected to some class of $\left(A, \lambda_{n}\right)$ summability methods in such a way that a formal series $\sum_{m=0}^{\infty} c_{m}$ is summable if and only if it is $\left(A, \lambda_{n}\right)$ summable and the partial sums defining summability method with $\lambda_{m} c_{m}$ instead of $c_{m}$ are $o\left(\lambda_{n}\right)$. We thus prove that the Ingham summability method is connected in this sense with $(A, \log n)$ method. It was shown in [11] that this pattern holds also for the Cesàro summability methods $(C, \theta)$ with $\theta>-1$ which are proved to be connected to $(A, n)$ method. In the same paper we exploited the connection of Cesàro summation method with the multiplicative functions on permutations to obtain an analog of the Theorem 1.3 providing the asymptotic estimate of the mean value of the multiplicative function on permutations.

\section{Proofs}

Let us start by introducing notations that will be used later in the paper. We will denote by $\Psi(x)$ the Chebyshev's function

$$
\Psi(x)=\sum_{m \leqslant x} \Lambda(m)
$$

where $\Lambda(m)$ - Mangoldt's function. We will also denote

$$
\Delta(x, y)=\Psi(y)-\Psi(x)-(y-x) .
$$

Later we will need an upper bound estimate of $\Delta(x, y)$ which we formulate as the next theorem. In fact much stronger estimate is known (see [5]). However we formulate the weakest estimate that we know to be sufficient for our proof of Theorem 2.5. 
Theorem $2.1([5])$. Suppose $c>0$ is a fixed constant. There exists a constant $\eta$ satisfying condition $0<\eta<1$ such that

$$
\Delta(x, x+h) \ll \frac{h}{\log x}, \quad \text { when } h \geqslant c x^{\eta},
$$

for $x \geqslant 2$, the constant in symbol $\ll i$ is absolute, depending only on $c$ and $\eta$.

For any $t>0$ we define a positive multiplicative function

$$
f_{t}(m)=\sum_{d \mid m} \frac{\mu(d)}{d^{t}}=\prod_{p \mid m}\left(1-\frac{1}{p^{t}}\right)>0,
$$

where $\mu(d)$ is Möbius function. The Dirichlet generating series of $f_{t}(m)$ is

$$
L_{t}(s)=\sum_{m=1}^{\infty} \frac{f_{t}(m)}{m^{s}}=\sum_{m=1}^{\infty} \frac{1}{m^{s}} \sum_{m=1}^{\infty} \frac{\mu(d)}{d^{s+t}}=\frac{\zeta(s)}{\zeta(s+t)},
$$

where $\zeta(s)=\sum_{m=1}^{\infty} m^{-s}$ is the Riemann Zeta function. We will denote the partial sums of $f_{t}(m)$ as

$$
F_{t}(x)=\sum_{1 \leqslant m \leqslant x} f_{t}(m), \quad \text { for } \quad x \geqslant 1 .
$$

Later we will need the estimates of the various sums involving $f_{t}(m)$, which we formulate as the following lemma.

Lemma 2.2. For any $x>1$ and $t>0$ we have

$$
\begin{gathered}
\sum_{m \leqslant x} \frac{f_{t}(m)}{m} \ll 1+t \log x \\
F_{t}(x)=\frac{x}{\zeta(1+t)}+O\left(x^{1-t}\right)+O\left(\sum_{d \leqslant x} \frac{1}{d^{t}}\right), \\
\sum_{m \leqslant x} \frac{f_{t}(m)}{m}=\sum_{d \leqslant x} \frac{\mu(d)}{d^{1+t}} \log \frac{x}{d}+O(1), \\
F_{t}(x)-F_{t}\left(\frac{x}{2}\right) \ll x\left(\frac{1}{\log x}+t\right),
\end{gathered}
$$

for $k \geqslant 2$ we have

$$
\int_{0}^{\infty} F_{t}(x)\left(\frac{1}{k^{t}}-\frac{1}{(k+1)^{t}}\right) d t=x \int_{0}^{\infty}\left(\frac{1}{k^{t}}-\frac{1}{(k+1)^{t}}\right) \frac{d t}{\zeta(1+t)}+O\left(\frac{x}{k \log ^{2} x k}\right) .
$$


Proof. The estimates of the lemma are trivial if $x \leqslant 3$, therefore throughout the proof we will assume that $x>3$. Recalling the formula for the Dirichlet generating function (13) of $f_{t}(m)$ we obtain

$$
\sum_{m \leqslant x} \frac{f_{t}(m)}{m} \leqslant e \sum_{m \leqslant x} \frac{f_{t}(m)}{m^{1+\frac{1}{\log x}}} \leqslant e \frac{\zeta\left(1+\frac{1}{\log x}\right)}{\zeta\left(1+\frac{1}{\log x}+t\right)} \ll 1+t \log x,
$$

since $\frac{1}{u-1}<\zeta(u)<\frac{u}{u-1}$ for any $u>1$. This proves (14).

To prove the next two estimates we replace $f_{t}(m)$ by a sum $\sum_{d \mid m} \mu(d) d^{-t}$. This way we obtain

$$
\begin{aligned}
F_{t}(x) & =\sum_{m \leqslant x} f_{t}(m)=\sum_{m \leqslant x} \sum_{d \mid m} \frac{\mu(d)}{d^{t}}=\sum_{d \leqslant x} \frac{\mu(d)}{d^{t}}\left[\frac{x}{d}\right] \\
& =\frac{x}{\zeta(1+t)}+O\left(x^{1-t}\right)+O\left(\sum_{d \leqslant x} \frac{1}{d^{t}}\right),
\end{aligned}
$$

here we estimated $\sum_{d>x} \mu(d) d^{-1-t} \ll x^{-t}$ by applying partial summation and utilizing the well-known fact that

$$
\left|\sum_{d \leqslant m} \frac{\mu(d)}{d}\right| \leqslant 1
$$

for all $m \geqslant 1$. This proves the estimate (15). In a similar way

$$
\begin{aligned}
\sum_{m \leqslant x} \frac{f_{t}(m)}{m} & =\sum_{m \leqslant x} \frac{1}{m} \sum_{d \mid m} \frac{\mu(d)}{d^{t}}=\sum_{d \leqslant x} \frac{\mu(d)}{d^{1+t}} \sum_{k \leqslant x / d} \frac{1}{k} \\
& =\sum_{d \leqslant x} \frac{\mu(d)}{d^{1+t}}\left(\log \frac{x}{d}-\gamma+O\left(\frac{d}{x}\right)\right)=\sum_{d \leqslant x} \frac{\mu(d)}{d^{1+t}} \log \frac{x}{d}+O(1)
\end{aligned}
$$

here we have used the estimate (19) of partial sums of $\mu(d) / d$. The estimate (16) is proved.

Differentiating by $s$ the Dirichlet series of $f_{t}(m)$ we get

$$
\begin{aligned}
\sum_{m=1}^{\infty} \frac{f_{t}(m) \log m}{m^{s}} & =-\frac{d}{d s} \frac{\zeta(s)}{\zeta(s+t)}=-\frac{\zeta(s)}{\zeta(s+t)}\left(\frac{\zeta^{\prime}(s)}{\zeta(s)}-\frac{\zeta^{\prime}(s+t)}{\zeta(s+t)}\right) \\
& =\sum_{m=1}^{\infty} \frac{f_{t}(m)}{m^{s}} \sum_{m=1}^{\infty} \frac{\Lambda(m)}{m^{s}}\left(1-\frac{1}{m^{t}}\right) .
\end{aligned}
$$

Equating the coefficients of $\frac{1}{m^{s}}$ in the above expression and summing by $m$ such that $m \leqslant x$ we get an identity

$$
\sum_{m \leqslant x} f_{t}(m) \log m=\sum_{k \ell \leqslant x} f_{t}(k) \Lambda(\ell)\left(1-\frac{1}{\ell^{t}}\right),
$$


therefore

$$
\begin{aligned}
F_{t}(x)-F_{t}\left(\frac{x}{2}\right) & \leqslant \frac{1}{\log \frac{x}{2}} \sum_{m \leqslant x} f_{t}(m) \log m \leqslant \frac{1}{\log \frac{x}{2}} \sum_{d \ell \leqslant x} f_{t}(d) \Lambda(\ell)\left(1-\frac{1}{\ell^{t}}\right) \\
& \leqslant \frac{1}{\log \frac{x}{2}} \sum_{d \leqslant x} f_{t}(d) \Psi\left(\frac{x}{d}\right) \ll \frac{x}{\log \frac{x}{2}} \sum_{d \leqslant x} \frac{f_{t}(d)}{d} \\
& \ll x\left(\frac{1}{\log x}+t\right),
\end{aligned}
$$

for $x \geqslant 3$. Here we have used the fact that $\Psi(x)=O(x)$ and applied the already proven estimate (14). This proves (17).

Applying the identity $f_{t}(m)=\sum_{d \mid m} \mu(d) d^{-t}$ we obtain

$$
\begin{gathered}
\int_{0}^{\infty} F_{t}(x)\left(\frac{1}{k^{t}}-\frac{1}{(k+1)^{t}}\right) d t=\sum_{m \leqslant x} \int_{0}^{\infty}\left(\frac{1}{k^{t}}-\frac{1}{(k+1)^{t}}\right)\left(\sum_{d \mid m} \frac{\mu(d)}{d^{t}}\right) d t \\
=\sum_{d \leqslant x} \mu(d)\left[\frac{x}{d}\right] \int_{0}^{\infty}\left(\frac{1}{k^{t}}-\frac{1}{(k+1)^{t}}\right) \frac{d t}{d^{t}} \\
=x \sum_{d \leqslant x} \frac{\mu(d)}{d} \int_{0}^{\infty}\left(\frac{1}{k^{t}}-\frac{1}{(k+1)^{t}}\right) \frac{d t}{d^{t}}+O\left(\sum_{d \leqslant x} \frac{\log \left(1+\frac{1}{k}\right)}{\log ^{2} d k}\right),
\end{gathered}
$$

for all $x \geqslant 1$. Using the estimate (19) of sums of $\mu(d) / d$ and applying partial summation we can estimate the tail of the series in the last expression as

$\left|\sum_{d>x} \frac{\mu(d)}{d} \int_{0}^{\infty}\left(\frac{1}{k^{t}}-\frac{1}{(k+1)^{t}}\right) \frac{d t}{d^{t}}\right| \leqslant 2 \int_{0}^{\infty}\left(\frac{1}{k^{t}}-\frac{1}{(k+1)^{t}}\right) \frac{d t}{x^{t}} \leqslant \frac{2}{k \log ^{2} k x}$.

Evaluating the sum inside the symbol $O(\ldots)$ in the previous estimate by means of inequality $\sum_{1 \leqslant d \leqslant x} \frac{1}{\log ^{2} d k} \ll \frac{x}{\log ^{2} x k}$ we complete the proof of the estimate (18).

Lemma 2.3. Suppose sequence $a_{k}$ is such that for any $v>1$

$$
\lim _{k \rightarrow \infty} \frac{|S(k)|}{k^{v}}=0
$$

then series $\sum_{m=1}^{\infty} \frac{a_{m}}{m^{v}}$ converges for all $v>1$.

Proof. Condition of the lemma implies by summation by parts that Dirichlet series

$$
\sum_{m=1}^{\infty} \frac{S(m)-S(m-1)}{m^{s}}
$$


converges for all $s>1$. Recalling the definition (6) of $S(m)$ we can express the difference $S(m)-S(m-1)$ as a sum of $a_{k} \log k$ in the following way

$$
S(m)-S(m-1)=\sum_{k \mid m} a_{k} \log k, \quad \text { for } \quad m \geqslant 1 .
$$

This means that if we multiply our convergent series (21) by an absolutely convergent series $\sum_{m=1}^{\infty} \frac{\mu(m)}{m^{s}}=1 / \zeta(s)$ then the resulting series

$$
\sum_{m=1}^{\infty} \frac{a_{m} \log m}{m^{s}}
$$

is also convergent for all $s>1$. This in its turn implies that if we integrate the above series with respect to $s$, then the resulting the series

$$
\sum_{m=1}^{\infty} \frac{a_{m}}{m^{s}}
$$

is also convergent for all $s>1$.

Lemma 2.4. Suppose sequence $a_{k}$ is such that

$$
\lim _{k \rightarrow \infty} \frac{|S(k)|}{k^{v}}=0
$$

for any $v>1$, then by Lemma 2.3 the function $g(s)=\sum_{m=1}^{\infty} \frac{a_{m}}{m^{s}}$ will be correctly defined for all $s>1$ and the identity

$$
\begin{aligned}
& \sum_{m=1}^{n} a_{m}\left[\frac{n}{m}\right]-n g\left(1+\frac{1}{\log n}\right)-\frac{S(n)}{\log n} \\
& \quad=\sum_{k=2}^{n-1} S(k) \int_{0}^{\infty}\left(\frac{F_{t}\left(\frac{n}{k}\right)}{k^{t}}-\frac{F_{t}\left(\frac{n}{k+1}\right)}{(k+1)^{t}}\right) d t-n \sum_{k=2}^{\infty} S(k) \int_{\sigma}^{\infty}\left(\frac{1}{k^{u}}-\frac{1}{(k+1)^{u}}\right) \frac{d u}{\zeta(u)},
\end{aligned}
$$

holds for all $n \geqslant 2$. Here we assume that $\sum_{k=2}^{1}(\ldots)=0$.

Proof. The Möbius inversion formula yields

$$
a_{m} \log m=\sum_{k \mid m} \mu\left(\frac{m}{k}\right)(S(k)-S(k-1)) \quad \text { when } \quad m \geqslant 1 .
$$

Inserting the above expression for $a_{k}$ into the righthand side of the identity (23) in the statement of our theorem, denoting

$$
\sigma=1+\frac{1}{\log n}
$$


and taking into account that $S(1)=S(0)=0$ we obtain

$$
\begin{aligned}
\sum_{m=1}^{n} a_{k}\left[\frac{n}{m}\right]-n g(\sigma)= & \sum_{m=2}^{n} a_{k}\left[\frac{n}{m}\right]-n \sum_{m=2}^{\infty} \frac{a_{m}}{m^{\sigma}} \\
= & \sum_{m=2}^{n}\left[\frac{n}{m}\right] \frac{1}{\log m} \sum_{k \mid m} \mu\left(\frac{m}{k}\right)(S(k)-S(k-1)) \\
& -n \sum_{m=2}^{\infty} \frac{1}{m^{\sigma} \log m} \sum_{k \mid m} \mu\left(\frac{m}{k}\right)(S(k)-S(k-1))
\end{aligned}
$$

Changing the order of summation of the two sums occurring in last expression we obtain

$$
\begin{aligned}
\sum_{m=1}^{n} a_{k}\left[\frac{n}{m}\right]-n g(\sigma)= & \sum_{k=2}^{n}(S(k)-S(k-1)) \sum_{\substack{m: 1 \leqslant m \leqslant n \\
k \mid m}}\left[\frac{n}{m}\right] \frac{\mu\left(\frac{m}{k}\right)}{\log m} \\
& -n \sum_{k=2}^{\infty}(S(k)-S(k-1)) \sum_{m: k \mid m} \frac{\mu\left(\frac{m}{k}\right)}{m^{\sigma} \log m}
\end{aligned}
$$

for $n \geqslant 2$. Let us show that the condition (26) imposed upon $|S(k)|$ guarantees that the exchanging of the order of summation is justified. Indeed, Lemma 2.3 guarantees the convergence of the series $\sum_{m=1}^{\infty} \frac{a_{m}}{m^{\sigma}}$, which means that

$$
\sum_{m=2}^{\infty} \frac{a_{m}}{m^{\sigma}}=\lim _{N \rightarrow \infty} \sum_{m=2}^{N} \frac{1}{m^{\sigma} \log m} \sum_{k \mid m} \mu\left(\frac{m}{k}\right)(S(k)-S(k-1))
$$

For any finite $N$ we can exchange the order of summation in the expression under the limit sign and fixing an integer $M \geqslant 3$ we obtain

$$
\begin{aligned}
\sum_{m=2}^{\infty} \frac{a_{m}}{m^{\sigma}}= & \lim _{N \rightarrow \infty} \sum_{k=2}^{N} \frac{S(k)-S(k-1)}{k^{\sigma}} \sum_{\ell \leqslant N / k} \frac{\mu(\ell)}{\ell^{\sigma} \log (k \ell)} \\
= & \sum_{k=2}^{M-1} \frac{S(k)-S(k-1)}{k^{\sigma}} \sum_{\ell=1}^{\infty} \frac{\mu(\ell)}{\ell^{\sigma} \log (k \ell)} \\
& +\lim _{N \rightarrow \infty} \sum_{k=M}^{N} \frac{S(k)-S(k-1)}{k^{\sigma}} \sum_{\ell \leqslant N / k} \frac{\mu(\ell)}{\ell^{\sigma} \log (k \ell)} \\
= & \sum_{k=2}^{M-1} \frac{S(k)-S(k-1)}{k^{\sigma}} \sum_{\ell=1}^{\infty} \frac{\mu(\ell)}{\ell^{\sigma} \log (k \ell)}+O\left(\frac{1}{M^{\sigma-\sigma^{\prime}}}\right),
\end{aligned}
$$


where $\sigma^{\prime}$ is a fixed number such that $1<\sigma^{\prime}<\sigma$. Indeed

$$
\sum_{k=M}^{N}(S(k)-S(k-1)) \frac{1}{k^{\sigma}} \sum_{\ell \leqslant N / k} \frac{\mu(\ell)}{\ell^{\sigma} \log (k \ell)}=\sum_{k=M}^{N}(S(k)-S(k-1)) \alpha_{k}
$$

with $\alpha_{k}=\frac{1}{k^{\sigma}} \sum_{\ell \leqslant N / k} \frac{\mu(\ell)}{\ell^{\sigma} \log (k \ell)}$, which are such that $\alpha_{k} \ll 1 / k^{\sigma}$ and

$$
\left|\alpha_{k}-\alpha_{k+1}\right| \ll \frac{1}{k^{\sigma+1}}+\frac{1}{N^{\sigma}}\left(\left[\frac{N}{k}\right]-\left[\frac{N}{k+1}\right]\right) .
$$

By condition of our lemma $S(n) \ll n^{\sigma^{\prime}}$. This by means of summation by parts and applying the above upper bound for $\left|\alpha_{k}-\alpha_{k-1}\right|$ leads to estimate

$$
\begin{aligned}
\sum_{k=M}^{N}(S(k)-S(k-1)) \alpha_{k} & \ll \frac{|S(M-1)|}{M^{\sigma}}+\frac{|S(N)|}{N^{\sigma}} \\
& +\sum_{k=M}^{N-1}|S(k)|\left(\frac{1}{k^{\sigma+1}}+\frac{1}{N^{\sigma}}\left(\left[\frac{N}{k}\right]-\left[\frac{N}{k+1}\right]\right)\right) \\
& \ll \frac{1}{M^{\sigma-\sigma^{\prime}}}+\frac{1}{N^{\sigma-\sigma^{\prime}}}
\end{aligned}
$$

whence we conclude that the upper limit of the above expression as $N \rightarrow \infty$ does not exceed $O\left(M^{-\left(\sigma-\sigma^{\prime}\right)}\right)$. This proves (25). Letting $M \rightarrow \infty$ in (25) we conclude that the change of summation in (24) is justified.

Let us express the quantities involving $\mu(d)$ in the identity (24) in terms of the function $f_{t}(m)$

$$
\begin{aligned}
\sum_{\substack{1 \leqslant m \leqslant n \\
k \mid m}}\left[\frac{n}{m}\right] \frac{\mu\left(\frac{m}{k}\right)}{\log m} & =\sum_{1 \leqslant d \leqslant \frac{n}{k}}\left[\frac{n}{k d}\right] \frac{\mu(d)}{\log k d}=\sum_{1 \leqslant m \leqslant \frac{n}{k}} \sum_{d \mid m} \frac{\mu(d)}{\log k d} \\
& =\sum_{1 \leqslant m \leqslant \frac{n}{k}} \sum_{d \mid m} \int_{0}^{\infty} \frac{\mu(d)}{(d k)^{t}} d t=\sum_{1 \leqslant m \leqslant \frac{n}{k}} \int_{0}^{\infty} \frac{1}{k^{t}} \prod_{p \mid m}\left(1-\frac{1}{p^{t}}\right) d t \\
& =\int_{0}^{\infty} \frac{F_{t}\left(\frac{n}{k}\right)}{k^{t}} d t .
\end{aligned}
$$

In a similar fashion we obtain

$$
\sum_{k \mid m} \frac{\mu\left(\frac{m}{k}\right)}{m^{\sigma} \log m}=\sum_{d=1}^{\infty} \frac{\mu(d)}{k^{\sigma} d^{\sigma} \log k d}=\int_{\sigma}^{\infty} \frac{d u}{k^{u} \zeta(u)} .
$$

Inserting the above expressions into (24) and using summation by parts in the resulting identities we complete the proof of the lemma. 
The estimate provided by the following theorem is a crucial part of our argument that will enable us to obtain the results stated in the introduction.

Theorem 2.5. Suppose sequence $a_{k}$ is such that for any $v>1$

$$
\lim _{k \rightarrow \infty} \frac{|S(k)|}{k^{v}}=0
$$

then the function $g(v)=\sum_{m=1}^{\infty} \frac{a_{m}}{m^{v}}$ is correctly defined for all $v>1$ and for $n \geqslant 2$ we have

$$
\left|\sum_{m=1}^{n} a_{k}\left[\frac{n}{m}\right]-n g\left(1+\frac{1}{\log n}\right)\right| \ll \sum_{k=2}^{n} c_{n, k}|S(k)|+\frac{n}{\log n} \sum_{k=n}^{\infty} \frac{|S(k)|}{k^{2+1 / \log n} \log k},
$$

where $c_{n, k}$ are non-negative real constants that satisfy the condition

$$
\sum_{k=2}^{n-1} c_{n, k} k(\log k)^{\varepsilon} \leqslant C(\varepsilon) n(\log n)^{\varepsilon-1}
$$

for any $0<\varepsilon \leqslant 1$, where $C(\varepsilon)>0$ is a constant which depends on $\varepsilon$ only. Moreover

$$
c_{n, k}=o(n), \quad \text { as } \quad n \rightarrow \infty
$$

for any fixed $k$.

Proof. Let us denote

$$
R_{n}=\sum_{m=1}^{n} a_{k}\left[\frac{n}{m}\right]-n g\left(1+\frac{1}{\log n}\right)-\frac{S(n)}{\log n}
$$

We will prove the theorem by estimating the quantities involved in the right hand side of identity (23) expressing $R_{n}$ in terms of quantities involving sums of $f_{t}(m)$. Throughout the proof we will denote

$$
\sigma=1+\frac{1}{\log n}
$$

Applying inequality $\zeta(u)>\frac{1}{u-1}$, which is true for all $u>1$, we obtain

$$
\begin{aligned}
\int_{1}^{\sigma}\left(\frac{1}{k^{u}}-\frac{1}{(k+1)^{u}}\right) \frac{d u}{\zeta(u)} & <\int_{1}^{\sigma} \frac{u-1}{k^{u}}\left(1-e^{-u \log \left(1+\frac{1}{k}\right)}\right) d u \\
& <\frac{\sigma}{k^{2}} \int_{0}^{\sigma-1} u d u=\frac{\sigma}{2 k^{2} \log ^{2} n} .
\end{aligned}
$$


For $k \geqslant n$ we have

$$
\int_{\sigma}^{\infty}\left(\frac{1}{k^{u}}-\frac{1}{(k+1)^{u}}\right) \frac{d u}{\zeta(u)} \ll \frac{1}{k^{\sigma+1} \log n \log k} .
$$

Putting $x=\frac{n}{k+1}$ in (18) we obtain

$$
\int_{0}^{\infty} F_{t}\left(\frac{n}{k+1}\right)\left(\frac{1}{k^{t}}-\frac{1}{(k+1)^{t}}\right) d t=\frac{n}{k+1} \int_{0}^{\infty}\left(\frac{1}{k^{t}}-\frac{1}{(k+1)^{t}}\right) \frac{d t}{\zeta(1+t)}+O\left(\frac{n}{k^{2} \log ^{2} n}\right) .
$$

Let us now use the above estimate together with (31) and (32) to further simplify the expression of $R_{n}$.

$$
\begin{array}{r}
R_{n}=\sum_{k=2}^{n-1} S(k)\left[\int_{0}^{\infty} \frac{F_{t}\left(\frac{n}{k}\right)-F_{t}\left(\frac{n}{k+1}\right)}{k^{t}} d t-\frac{n}{k(k+1)} \int_{0}^{\infty} \frac{d t}{k^{t} \zeta(1+t)}\right] \\
+O\left(\frac{n}{\log n} \sum_{k=n}^{\infty} \frac{|S(k)|}{k^{\sigma+1} \log k}+\frac{n}{\log ^{2} n} \sum_{k=2}^{n} \frac{|S(k)|}{k^{2}}\right) .
\end{array}
$$

Suppose $\sqrt{n} \leqslant k \leqslant n-1$, then $\frac{n}{k}-\frac{n}{k+1}=\frac{n}{k(k+1)}<1$. This means that there can be only one natural number between $\frac{n}{k}$ and $\frac{n}{k+1}$. In which case, if there exists such $m$ that $\frac{n}{k} \geqslant m>\frac{n}{k+1}$, we have $k \leqslant \frac{n}{m}$ and $k+1>\frac{n}{m}$. This means that $\left[\frac{n}{m}\right] \geqslant k>\left[\frac{n}{m}\right]-1$. Which implies that $k=\left[\frac{n}{m}\right]$. And conversely, for $k=\left[\frac{n}{m}\right]$, we have $\frac{n}{k} \geqslant m>\frac{n}{k+1}$. Thus the only natural numbers $k$ in the interval $\sqrt{n} \leqslant k \leqslant n-1$ such that the interval $\left[\frac{n}{k}, \frac{n}{k+1}\right)$ contains some natural number $m$ and subsequently $F_{t}\left(\frac{n}{k}\right)-F_{t}\left(\frac{n}{k+1}\right)=f_{t}(m)$ are of the form $k=[n / m]$. This observation allows us to further simplify the estimate of the sum over $k>\sqrt{n}$ in the estimate of $R_{n}$ and obtain

$$
\begin{aligned}
\left|R_{n}\right| \leqslant & \sum_{2 \leqslant k<\sqrt{n}}|S(k)|\left|\int_{0}^{\infty} \frac{F_{t}\left(\frac{n}{k}\right)-F_{t}\left(\frac{n}{k+1}\right)}{k^{t}} d t-\frac{n}{k(k+1)} \int_{0}^{\infty} \frac{d t}{k^{t} \zeta(1+t)}\right| \\
& +\sum_{2 \leqslant m \leqslant \sqrt{n}}\left|S\left(\left[\frac{n}{m}\right]\right)\right| \int_{0}^{\infty} \frac{f_{t}(m)}{[n / m]^{t}} d t+O\left(\frac{n}{\log n} \sum_{k=n}^{\infty} \frac{|S(k)|}{k^{\sigma+1} \log k}+\frac{n}{\log ^{2} n} \sum_{k=2}^{n} \frac{|S(k)|}{k^{2}}\right) .
\end{aligned}
$$

Thus the inequality (27) holds if for $k \leqslant \sqrt{n}$ we put

$$
c_{n, k}=\left|\int_{0}^{\infty} \frac{F_{t}\left(\frac{n}{k}\right)-F_{t}\left(\frac{n}{k+1}\right)}{k^{t}} d t-\frac{n}{k(k+1)} \int_{0}^{\infty} \frac{d t}{k^{t} \zeta(1+t)}\right|+\frac{n}{k^{2} \log ^{2} n}
$$


and for $k>\sqrt{n}$ define

$c_{n, k}= \begin{cases}\frac{n}{k^{2} \log ^{2} n}, & \text { if } \sqrt{n}<k \leqslant n-1 \text { and } k \neq[n / m] \text { for any } m \leqslant \sqrt{n}, \\ \int_{0}^{\infty} \frac{f_{t}(m)}{[n / m]^{t}} d t+\frac{n}{k^{2} \log ^{2} n}, & \text { if } \sqrt{n}<k \leqslant n-1 \text { and } k=[n / m] \text { for some } m \leqslant \sqrt{n} \\ \frac{n}{\log n}, & \text { if } k=n .\end{cases}$

Plugging the estimate (15) of $F_{t}(x)$ into our definition of $c_{n, k}$ in (33) after some easy calculations we conclude that for fixed $k$ we have $c_{n, k}=o(n)$.

It remains to check that thus defined $c_{n, k}$ satisfy the condition (28) for any fixed $0<\varepsilon \leqslant 1$. We will do this by splitting the sum involving $c_{n, k}$ into three parts

$$
\begin{aligned}
\sum_{2 \leqslant k \leqslant n-1} c_{n, k} k(\log k)^{\varepsilon} & =\sum_{k \leqslant n^{\alpha}} c_{n, k} k(\log k)^{\varepsilon}+\sum_{n^{\alpha}<k<\sqrt{n}} c_{n, k} k(\log k)^{\varepsilon}+\sum_{\sqrt{n} \leqslant k \leqslant n-1} c_{n, k} k(\log k)^{\varepsilon} \\
& =: K_{1}+K_{2}+K_{3} .
\end{aligned}
$$

Here and further $0<\alpha<1 / 2$ will be fixed arbitrarily chosen number, upon which we will later impose additional upper bound conditions.

The case of estimating $K_{3}$ the sum of $c_{n, k}$ over interval $\sqrt{n} \leqslant k \leqslant n-1$ is the easiest. By our expression (34) for $c_{n, k}$ belonging to this interval we have

$$
\begin{aligned}
K_{3} & =\sum_{\sqrt{n} \leqslant k \leqslant n-1} c_{n, k} k(\log k)^{\varepsilon} \\
& \ll \sum_{m \leqslant \sqrt{n}} \frac{n}{m}\left(\log \frac{n}{m}\right)^{\varepsilon} \int_{0}^{\infty} \frac{f_{t}(m)}{[n / m]^{t}} d t+\sum_{\sqrt{n} \leqslant k \leqslant n-1} \frac{n}{k^{2} \log ^{2} n} k(\log k)^{\varepsilon} \\
& \ll n(\log n)^{\varepsilon} \int_{0}^{\infty} \frac{1}{n^{t / 2}} \sum_{m \leqslant \sqrt{n}} \frac{f_{t}(m)}{m} d t+n(\log n)^{\varepsilon-1} \\
& \ll n(\log n)^{\varepsilon} \int_{0}^{\infty} \frac{1+t \log n}{n^{t / 2}} d t+n(\log n)^{\varepsilon-1} \ll n(\log n)^{\varepsilon-1}
\end{aligned}
$$

Here we have used the upper bound for sum $\sum_{m \leqslant x} \frac{f_{t}(m)}{m}$ provided by estimate (14) of Lemma2.2. 
Let us now estimate $K_{2}$ - the sum over interval $n^{\alpha}<m<\sqrt{n}$. We have

$$
\begin{aligned}
K_{2}= & \sum_{n^{\alpha}<k<\sqrt{n}} c_{n, k} k(\log k)^{\varepsilon} \\
\ll & (\log n)^{\varepsilon} \sum_{n^{\alpha}<k<\sqrt{n}} k \int_{0}^{\infty} \frac{F_{t}\left(\frac{n}{k}\right)-F_{t}\left(\frac{n}{k+1}\right)}{k^{t}} d t \\
& +(\log n)^{\varepsilon} \sum_{n^{\alpha}<k<\sqrt{n}} \frac{n}{k} \int_{0}^{\infty} \frac{d t}{k^{t} \zeta(1+t)}+\sum_{n^{\alpha}<k<\sqrt{n}} \frac{n}{k^{2} \log ^{2} n} k(\log k)^{\varepsilon}
\end{aligned}
$$

The second and the third sum in the last estimate are clearly $O\left(n(\log n)^{\varepsilon-1}\right)$. The first sum in the above upper bound can be estimated as

$$
\begin{aligned}
\sum_{n^{\alpha}<k<\sqrt{n}} k \int_{0}^{\infty} \frac{F_{t}\left(\frac{n}{k}\right)-F_{t}\left(\frac{n}{k+1}\right)}{k^{t}} d t & \leqslant \int_{0}^{\infty} \frac{1}{n^{\alpha t}} \sum_{n^{\alpha}<k<\sqrt{n}} k\left(F_{t}\left(\frac{n}{k}\right)-F_{t}\left(\frac{n}{k+1}\right)\right) d t \\
& \leqslant n \int_{0}^{\infty} \frac{1}{n^{\alpha t}} \sum_{n^{\alpha}<k<\sqrt{n}} \frac{k}{n} \sum_{\frac{n}{k+1}<m \leqslant \frac{n}{k}} f_{t}(m) d t \\
& \leqslant n \int_{0}^{\infty} \frac{1}{n^{\alpha t}} \sum_{n^{\alpha}<k<\sqrt{n}} \sum_{\frac{n}{k+1}<m \leqslant \frac{n}{k}} \frac{f_{t}(m)}{m} d t \\
& \leqslant n \int_{0}^{\infty} \frac{1}{n^{\alpha t}} \sum_{m=1}^{n} \frac{f_{t}(m)}{m} d t .
\end{aligned}
$$

We can use the upper bound for sum $\sum_{m=1}^{n} \frac{f_{t}(m)}{m}$ as provided in Lemma 2.2 to further estimate

$$
\sum_{n^{\alpha}<k<\sqrt{n}} k \int_{0}^{\infty} \frac{F_{t}\left(\frac{n}{k}\right)-F_{t}\left(\frac{n}{k+1}\right)}{k^{t}} d t \ll n \int_{0}^{\infty} \frac{1+t \log n}{n^{\alpha t}} d t \ll \frac{n}{\log n} .
$$

Inserting this estimate into (36) we get

$$
K_{2}=\sum_{n^{\alpha}<k<\sqrt{n}} c_{n, k} k(\log k)^{\varepsilon} \ll n(\log n)^{\varepsilon-1} .
$$

The case of the $K_{1}$, the sum over $k$ such that $k \leqslant n^{\alpha}$ is more complicated. We will prove that it is also $O\left(n(\log n)^{\varepsilon-1}\right)$. The reason of considering separately part $k \geqslant n^{\alpha}$ is that when $k \leqslant n^{\alpha}$ the gap between numbers $n / k$ and $n /(k+1)$ 
will be large enough to apply Theorem 2.1 to estimate the quantity $F_{t}(n / k)-$ $F_{t}(n /(k+1))$. We have

$$
-\frac{d}{d s} L_{t}(s)=L_{t}(s)\left(-\frac{\zeta^{\prime}(s)}{\zeta(s)}\right)-\frac{d}{d t} L_{t}(s)
$$

which means that

$$
f_{t}(m) \log m=\sum_{d l=m} f_{t}(d) \Lambda(l)+\frac{d}{d t} f_{t}(m) .
$$

Hence for $k \leqslant \sqrt{n}$

$$
\begin{aligned}
F_{t}\left(\frac{n}{k}\right)-F_{t}\left(\frac{n}{k+1}\right)=\sum_{\frac{n}{k+1}<m \leqslant \frac{n}{k}} f_{t}(m) \\
=\frac{1}{\log \frac{n}{k}} \sum_{\frac{n}{k+1}<m \leqslant \frac{n}{k}} f_{t}(m) \log m+\frac{1}{\log \frac{n}{k}} \sum_{\frac{n}{k+1}<m \leqslant \frac{n}{k}} f_{t}(m) \log \frac{n}{k m} \\
=\frac{1}{\log \frac{n}{k}} \sum_{\frac{n}{k+1}<m \leqslant \frac{n}{k}} f_{t}(m) \log m+O\left(\frac{1}{k \log n}\left(F_{t}\left(\frac{n}{k}\right)-F_{t}\left(\frac{n}{k+1}\right)\right)\right) \\
=\frac{1}{\log \frac{n}{k}} \sum_{m \leqslant \frac{n}{k}} f_{t}(m)\left(\Psi\left(\frac{n}{k m}\right)-\Psi\left(\frac{n}{(k+1) m}\right)\right) \\
\quad+O\left(\frac{1}{\log n} \frac{d}{d t}\left(F_{t}\left(\frac{n}{k}\right)-F_{t}\left(\frac{n}{k+1}\right)\right)\right)+O\left(\frac{n}{k^{3} \log n}\right) .
\end{aligned}
$$

Plugging our expression (40) into our formula for $c_{n, k}$ we have

$$
\begin{aligned}
K_{1}= & \sum_{2 \leqslant k \leqslant n^{\alpha}} k(\log k)^{\varepsilon}\left|\int_{0}^{\infty} \frac{F_{t}\left(\frac{n}{k}\right)-F_{t}\left(\frac{n}{k+1}\right)}{k^{t}} d t-\frac{n}{k(k+1)} \int_{0}^{\infty} \frac{d t}{k^{t} \zeta(1+t)}\right|+O\left(n(\log n)^{\varepsilon-1}\right) \\
\ll & \sum_{k \leqslant n^{\alpha}} k(\log k)^{\varepsilon} \mid \int_{0}^{\infty} \frac{1}{\log \frac{n}{k}} \sum_{m \leqslant \frac{n}{k}} f_{t}(m)\left(\Psi\left(\frac{n}{k m}\right)-\Psi\left(\frac{n}{(k+1) m}\right)\right) \frac{d t}{k^{t}} \\
& -\frac{n}{k(k+1)} \int_{0}^{\infty} \frac{d t}{k^{t} \zeta(1+t)} \mid \\
& +\frac{1}{\log n} \sum_{2 \leqslant k<\sqrt{n}} k(\log k)^{\varepsilon} \int_{0}^{\infty} \frac{1}{k^{t}} \frac{d}{d t}\left(F_{t}\left(\frac{n}{k}\right)-F_{t}\left(\frac{n}{k+1}\right)\right) d t+O\left(n(\log n)^{\varepsilon-1}\right)
\end{aligned}
$$


Note that $\frac{d f_{t}(m)}{d t}>0$ for all $t>0$. Therefore the sum involving derivative $\frac{d}{d t}\left(F_{t}\left(\frac{n}{k}\right)-F_{t}\left(\frac{n}{k+1}\right)\right)>0$ can be estimated by applying partial integration

$$
\begin{aligned}
\frac{1}{\log n} & \sum_{2 \leqslant k<\sqrt{n}} k(\log k)^{\varepsilon} \int_{0}^{\infty} \frac{1}{k^{t}} \frac{d}{d t}\left(F_{t}\left(\frac{n}{k}\right)-F_{t}\left(\frac{n}{k+1}\right)\right) d t \\
& \ll \frac{1}{\log n} \sum_{2 \leqslant k<\sqrt{n}} k(\log k)^{\varepsilon+1} \int_{0}^{\infty}\left(F_{t}\left(\frac{n}{k}\right)-F_{t}\left(\frac{n}{k+1}\right)\right) \frac{d t}{k^{t}} \\
& \ll \frac{1}{\log n} \sum_{1 \leqslant s \leqslant \frac{\log n}{2 \log 2}} \sum_{2^{s} \leqslant k<2^{s+1}} k(\log k)^{\varepsilon+1} \int_{0}^{\infty}\left(F_{t}\left(\frac{n}{k}\right)-F_{t}\left(\frac{n}{k+1}\right)\right) \frac{d t}{k^{t}} \\
& \ll \frac{1}{\log n} \sum_{1 \leqslant s \leqslant \frac{\log n}{2 \log 2}} 2^{s} s^{\varepsilon+1} \int_{0}^{\infty}\left(F_{t}\left(\frac{n}{2^{s}}\right)-F_{t}\left(\frac{n}{2^{s+1}}\right)\right) \frac{d t}{2^{s t}}
\end{aligned}
$$

Applying now (17) to estimate $F_{t}\left(\frac{n}{2^{s}}\right)-F_{t}\left(\frac{n}{2^{s+1}}\right)$ we get

$$
\begin{aligned}
\frac{1}{\log n} & \sum_{2 \leqslant k<\sqrt{n}} k(\log k)^{\varepsilon} \int_{0}^{\infty} \frac{1}{k^{t}} \frac{d}{d t}\left(F_{t}\left(\frac{n}{k}\right)-F_{t}\left(\frac{n}{k+1}\right)\right) d t \\
& \ll \frac{n}{\log n} \sum_{1 \leqslant s \leqslant \frac{\log n}{2 \log 2}} s^{\varepsilon+1} \int_{0}^{\infty}\left(t+\frac{1}{\log n}\right) \frac{d t}{2^{s t}} \\
& \ll n(\log n)^{\varepsilon-1}
\end{aligned}
$$

Applying this estimate to continue the evaluation of $K_{1}$ in (41) we obtain

$$
\begin{aligned}
K_{1} \ll & \sum_{k \leqslant n^{\alpha}} k(\log k)^{\varepsilon}\left|\int_{0}^{\infty} \frac{1}{\log \frac{n}{k}} \sum_{m \leqslant \frac{n}{k}} f_{t}(m) \Delta\left(\frac{n}{k m}, \frac{n}{(k+1) m}\right) \frac{d t}{k^{t}}\right| \\
& +n \sum_{k \leqslant n^{\alpha}} \frac{(\log k)^{\varepsilon}}{(k+1)}\left|\int_{0}^{\infty}\left(\frac{1}{\zeta(1+t)}-\frac{1}{\log \frac{n}{k}} \sum_{m \leqslant \frac{n}{k}} \frac{f_{t}(m)}{m}\right) \frac{d t}{k^{t}}\right|+O\left(n(\log n)^{\varepsilon-1}\right) .
\end{aligned}
$$

The last sum in the above equation can be estimated applying estimate (16) of Lemma 2.2 as

$$
\int_{0}^{\infty}\left(\frac{1}{\zeta(1+t)}-\frac{1}{\log \frac{n}{k}} \sum_{m \leqslant \frac{n}{k}} \frac{f_{t}(m)}{m}\right) \frac{d t}{k^{t}} \ll \frac{1}{\log n \log k}
$$


for $k \leqslant \sqrt{n}$. This gives us

$$
K_{1}=\sum_{2 \leqslant k \leqslant n^{\alpha}} c_{n, k} k(\log k)^{\varepsilon} \ll D+O\left(n(\log n)^{\varepsilon-1}\right) .
$$

Here

$$
D=\sum_{k \leqslant n^{\alpha}} k(\log k)^{\varepsilon}\left|\int_{0}^{\infty} \frac{1}{\log \frac{n}{k}} \sum_{m \leqslant \frac{n}{k}} f_{t}(m) \Delta\left(\frac{n}{k m}, \frac{n}{(k+1) m}\right) \frac{d t}{k^{t}}\right| \leqslant D_{1}+D_{2}
$$

where

$$
D_{1}=\sum_{k \leqslant n^{\alpha}} k(\log k)^{\varepsilon}\left|\int_{0}^{\infty} \frac{1}{\log \frac{n}{k}} \sum_{\frac{n}{k^{1+\delta}} \leqslant m \leqslant \frac{n}{k}} f_{t}(m) \Delta\left(\frac{n}{k m}, \frac{n}{(k+1) m}\right) \frac{d t}{k^{t}}\right|
$$

and

$$
D_{2}=\sum_{k \leqslant n^{\alpha}} k(\log k)^{\varepsilon} \int_{0}^{\infty} \frac{1}{\log \frac{n}{k}} \sum_{m \leqslant \frac{n}{k^{1+\delta}}} f_{t}(m)\left|\Delta\left(\frac{n}{k m}, \frac{n}{(k+1) m}\right)\right| \frac{d t}{k^{t}},
$$

here $\delta>0$ - fixed, such that $\alpha(1+\delta)<1$.

Then

$$
\begin{aligned}
D_{1} \ll & \frac{1}{\log n} \sum_{k \leqslant n^{\alpha}} k(\log k)^{\varepsilon} \int_{0}^{\infty} \sum_{\frac{n}{k^{1+\delta}} \leqslant m \leqslant \frac{n}{k}} f_{t}(m)\left(\Psi\left(\frac{n}{k m}\right)-\Psi\left(\frac{n}{(k+1) m}\right)\right) \frac{d t}{k^{t}} \\
& +\frac{n}{\log n} \sum_{k \leqslant n^{\alpha}} \frac{(\log k)^{\varepsilon}}{k} \int_{0}^{\infty} \sum_{\frac{n}{k^{1+\delta} \leqslant m \leqslant \frac{n}{k}}} \frac{f_{t}(m)}{m} \frac{d t}{k^{t}}=: J_{1}+J_{2} .
\end{aligned}
$$

Changing the order of summation in $J_{1}$ we get

$$
\begin{aligned}
J_{1} & =\frac{1}{\log n} \sum_{k \leqslant n^{\alpha}} k(\log k)^{\varepsilon} \int_{0}^{\infty} \sum_{\frac{n}{k^{1+\delta} \leqslant m \leqslant \frac{n}{k}}} f_{t}(m)\left(\Psi\left(\frac{n}{k m}\right)-\Psi\left(\frac{n}{(k+1) m}\right)\right) \frac{d t}{k^{t}} \\
& =\frac{1}{\log n} \sum_{n^{1-\alpha(1+\delta) \leqslant m \leqslant n / 2}} \int_{0}^{\infty} f_{t}(m) \sum_{\frac{1}{\left.\frac{1}{m}\right)^{1+\delta} \leqslant k \leqslant \frac{n}{m}}} k(\log k)^{\varepsilon}\left(\Psi\left(\frac{n}{k m}\right)-\Psi\left(\frac{n}{(k+1) m}\right)\right) \frac{d t}{k^{t}} \\
& \leqslant \frac{1}{\log n} \sum_{n^{1-\alpha(1+\delta) \leqslant m \leqslant n / 2}}\left(\log \frac{n}{m}\right)^{\varepsilon} \int_{0}^{\infty} f_{t}(m) \sum_{k \leqslant \frac{n}{m}} k\left(\Psi\left(\frac{n}{k m}\right)-\Psi\left(\frac{n}{(k+1) m}\right)\right)\left(\frac{m}{n}\right)^{\frac{t}{1+\delta}} d t
\end{aligned}
$$


Since for any $x \geqslant 1$

$$
\sum_{k=1}^{\infty} k\left(\Psi\left(\frac{x}{k}\right)-\Psi\left(\frac{x}{k+1}\right)\right)=\sum_{k=1}^{\infty} \Psi\left(\frac{x}{k}\right) \ll x \sum_{k=1}^{[x]} \frac{1}{k} \ll x \log x
$$

we get

$$
\begin{aligned}
J_{1} & \ll \frac{n}{\log n} \sum_{n^{1-\alpha(1+\delta) \leqslant m \leqslant n / 2 ~}}\left(\log \frac{n}{m}\right)^{\varepsilon+1} \int_{0}^{\infty} \frac{f_{t}(m)}{m}\left(\frac{m}{n}\right)^{\frac{t}{1+\delta}} d t \\
& \ll \frac{n}{\log n} \sum_{1 \leqslant s \leqslant(1-\alpha(1+\delta)) \log _{2} n \frac{n}{2^{s+1}<m \leqslant \frac{n}{2^{s}}}}\left(\log \frac{n}{m}\right)^{\varepsilon+1} \int_{0}^{\infty} \frac{f_{t}(m)}{m}\left(\frac{m}{n}\right)^{\frac{t}{1+\delta}} d t \\
& \ll \frac{1}{\log n} \sum_{1 \leqslant s \leqslant(1-\alpha(1+\delta)) \log _{2} n} s^{\varepsilon+1} 2^{s} \int_{0}^{\infty}\left(F_{t}\left(\frac{n}{2^{s}}\right)-F_{t}\left(\frac{n}{2^{s+1}}\right)\right) 2^{-\frac{s t}{1+\delta}} d t \\
& \ll \frac{n}{\log n} \sum_{1 \leqslant s \leqslant(1-\alpha(1+\delta)) \log _{2} n} s^{\varepsilon+1} \int_{0}^{\infty}\left(\frac{1}{\log n}+t\right) 2^{-\frac{s t}{1+\delta}} d t \ll n(\log n)^{\varepsilon-1} .
\end{aligned}
$$

We estimate $J_{2}$ in a similar way as $J_{1}$. First changing summation we get

$$
\begin{aligned}
J_{2} & =\frac{n}{\log n} \sum_{n^{1-\alpha(1+\delta) \leqslant m \leqslant n / 2}} \int_{0}^{\infty} f_{t}(m) \sum_{\frac{1}{\left.\frac{n}{m}\right)^{1+\delta} \leqslant k \leqslant \frac{n}{m}}} \frac{(\log k)^{\varepsilon}}{k^{1+t}} d t \\
& \leqslant \frac{n}{\log n} \sum_{n^{1-\alpha(1+\delta) \leqslant m \leqslant n / 2}}\left(\log \frac{n}{m}\right)^{\varepsilon+1} \int_{0}^{\infty} \frac{f_{t}(m)}{m}\left(\frac{m}{n}\right)^{\frac{t}{1+\delta}} d t .
\end{aligned}
$$

The last sum has already been estimated before while evaluating $J_{1}$ in (47), thus finally we get

$$
J_{2} \ll n(\log n)^{\varepsilon-1} .
$$

Our estimates of $J_{1}$ and $J_{2}$ implies that

$$
D_{1} \ll n(\log n)^{\varepsilon-1} .
$$

Let us now turn to estimating the sum $D_{2}$. Let us chose $\delta=1 /(1-\eta)$ where $\eta$ is the same as in formulation of Theorem 2.1 then

$$
\frac{n}{m k}-\frac{n}{m(k+1)} \geqslant\left(\frac{2}{3}\right)^{1-\eta}\left(\frac{n}{m(k+1)}\right)^{\eta}
$$


for $m \leqslant \frac{n}{k^{1+\delta}}$. Additionally let us assume that $\alpha>0$ is small enough to ensure that $\alpha(\delta+1)<1$. Then we can make use of Theorem 2.1 to evaluate

$$
\Delta\left(\frac{n}{k m}, \frac{n}{(k+1) m}\right) \ll \frac{n}{k^{2}} \frac{1}{\log \frac{n}{k m}} .
$$

Hence we obtain

$$
D_{2} \ll \frac{n}{\log n} \sum_{k \leqslant n^{\alpha}} \frac{(\log k)^{\varepsilon}}{k} \int_{0}^{\infty} \sum_{m \leqslant \frac{n}{k^{1+\delta}}} \frac{f_{t}(m)}{m \log \frac{n}{m k}} \frac{d t}{k^{t}}
$$

Changing the order of summation in the sum occurring in the last expression we get

$$
\begin{aligned}
D_{2} & \ll \frac{n}{\log n} \sum_{1 \leqslant m \leqslant n / 2}\left(\log \frac{n}{m}\right)^{\varepsilon-1} \int_{0}^{\infty} \frac{f_{t}(m)}{m} \sum_{2 \leqslant k \leqslant\left(\frac{n}{m}\right)^{\frac{1}{1+\delta}}} \frac{1}{k^{t+1}} d t \\
& \ll \frac{n}{\log n} \sum_{1 \leqslant s \leqslant \log _{2} \frac{n}{2}} \sum_{\frac{n}{2^{s+1}}<m \leqslant \frac{n}{2^{s}}}\left(\log \frac{n}{m}\right)^{\varepsilon-1} \int_{0}^{\infty} \frac{f_{t}(m)}{m} \sum_{2 \leqslant k \leqslant\left(\frac{n}{m}\right)^{\frac{1}{1+\delta}}} \frac{1}{k^{t+1}} d t \\
& \ll \frac{1}{\log n} \sum_{1 \leqslant s \leqslant \log _{2} \frac{n}{2}} s^{\varepsilon-1} 2^{s} \int_{0}^{\infty}\left(F_{t}\left(\frac{n}{2^{s}}\right)-F_{t}\left(\frac{n}{2^{s+1}}\right)\right) \sum_{2 \leqslant k \leqslant 2^{s+1}} \frac{1}{k^{t+1}} d t
\end{aligned}
$$

Applying here the estimate (17) for $F_{t}(x)-F_{t}(x / 2)$ with $x=n 2^{-s}$ we further estimate

$$
\begin{aligned}
D_{2} & \ll \frac{n}{\log n} \sum_{1 \leqslant s \leqslant \log _{2} \frac{n}{2}} s^{\varepsilon-1} \int_{0}^{\infty}\left(\frac{1}{\log \frac{n}{2^{s}}}+t\right) \sum_{2 \leqslant k \leqslant 2^{s+1}} \frac{1}{k^{t+1}} d t \\
& \ll \frac{n}{\log n} \sum_{1 \leqslant s \leqslant \log _{2} \frac{n}{2}} s^{\varepsilon-1}\left(\frac{1}{\log \frac{n}{2^{s}}} \sum_{2 \leqslant k \leqslant 2^{s+1}} \frac{1}{k \log k}+\sum_{2 \leqslant k \leqslant 2^{s+1}} \frac{1}{k(\log k)^{2}}\right) \\
& \ll n(\log n)^{\varepsilon-1} .
\end{aligned}
$$

Thus we have proved that

$$
K_{1}=\sum_{k \leqslant n^{\alpha}} c_{n, k} k(\log k)^{\varepsilon} \ll D_{1}+D_{2}+n(\log n)^{\varepsilon-1} \ll n(\log n)^{\varepsilon-1} .
$$

Our estimates of $K_{1}, K_{2}$ and $K_{3}$ allow us to evaluate the sum (35) as $O\left(n(\log n)^{\varepsilon-1}\right)$, which finally completes the proof of the theorem. 
Proof of Theorem 1.1. Plugging $S(n)=o(n \log n)$ into inequality (27) of Theorem 2.5 and making use of properties (28) and (29) of quantities $c_{n, k}$ we conclude that the right hand side of (27) is $o(n)$. Dividing both sides of thus obtained inequality by $n$ we complete the proof of the Theorem.

Lemma 2.6. Suppose $a_{k}$ is a sequence of complex numbers such that

$$
A(n)=\sum_{k \leqslant n} a_{k}\left[\frac{n}{k}\right]=C n+o(n),
$$

as $n \rightarrow \infty$, with some constant $C \in \mathbb{C}$.

Then

$$
S(n)=\sum_{k \leqslant n} a_{k}\left[\frac{n}{k}\right] \log k=o(n \log n),
$$

as $n \rightarrow \infty$.

Proof. The equality of $f(m)$ to the sum $\sum_{d \mid m} a_{d}$ is equivalent to identity

$$
U(s)=\sum_{m=1}^{\infty} \frac{f(m)}{m^{s}}=\sum_{m=1}^{\infty} \frac{1}{m^{s}} \sum_{m=1}^{\infty} \frac{a_{m}}{m^{s}}=\zeta(s) g(s) .
$$

Therefore

$$
\zeta(s) g^{\prime}(s)=(\zeta(s) g(s))^{\prime}-\zeta^{\prime}(s) g(s)=U^{\prime}(s)-\frac{\zeta^{\prime}(s)}{\zeta(s)} U(s)
$$

this identity corresponds to the equality of the coefficients of $m^{-s}$ of the corresponding Dirichlet series

$$
\sum_{k \mid m} a_{k} \log k=f(m) \log m-\sum_{k \ell=m} \Lambda(k) f(\ell)
$$

for all $m \geqslant 1$. Summing the above identity over all $m$ such that $m \leqslant n$ and recalling that $f(1)+f(2)+\cdots+f(k)=A(k)$ we get

$$
\begin{aligned}
S(n) & =\sum_{k=1}^{n} f(k) \log k-\sum_{k \ell \leqslant n} \Lambda(k) f(\ell) \\
& =\sum_{k=1}^{n}(A(k)-A(k-1)) \log k-\sum_{k \leqslant n} \Lambda(k) A\left(\frac{n}{k}\right) \\
& =A(n) \log n-\sum_{k=1}^{n} A(k) \log \left(1+\frac{1}{k}\right)-\sum_{k \leqslant n} \Lambda(k) A\left(\frac{n}{k}\right) .
\end{aligned}
$$


By condition of the lemma $A(n)=C n+o(n)$. Inserting this estimate into the above expression of $S(n)$ we get

$$
\begin{aligned}
S(n) & =A(n) \log n-\sum_{k \leqslant n} \Lambda(k) A\left(\frac{n}{k}\right)+O(n) \\
& =C n \log n-C n \sum_{k \leqslant n} \frac{\Lambda(k)}{k}+o(n \log n)=o(n \log n),
\end{aligned}
$$

where we have used the fact that $\sum_{k \leqslant n} \frac{\Lambda(k)}{k}=\log n+O(n)$.

The lemma is proved.

Proof of theorem 1.2 The sufficiency of the two conditions of the theorem for the existence of the limit of the sum (2) follows immediately from the Theorem 1.1 .

The necessity of the first condition of the theorem follows from Lemma 2.6 , The necessity of the second condition will follow if we note that function $g(s)$ can be represented as a fraction

$$
g(s)=\frac{\zeta(s) g(s)}{\zeta(s)}=\frac{\sum_{m=1}^{\infty} \frac{f(m)}{m^{s}}}{\sum_{m=1}^{\infty} \frac{1}{m^{s}}},
$$

where as before $f(m)=\sum_{d \mid m} a_{d}$. The partial sums of the coefficients of the Dirichlet series in the nominator satisfies $f(1)+f(2)+\cdots+f(n)=\sum_{k=1}^{n} a_{k}\left[\frac{n}{k}\right]=$ $C n+o(n)$, by our assumtion. Thus passing to the limit $s \downarrow 1$ we conclude that $\lim _{s \downarrow 1} g(s)=C$.

Proof of theorem 1.3 Once we are given the values of $f$ on prime numbers $p$ such that $p \leqslant n$ we can compute the value of function $f$ on any integer $m$ such that $m \leqslant n$. The numbers $f(p)$, with $p>n$ do not influence the value of the quantity

$$
\frac{1}{n} \sum_{m=1}^{n} f(m)
$$

therefore we will assume that $f(p)=1$ for $p>n$.

We have already noted that if $f(m)=\sum_{d \mid m} a_{d}$ then the Dirichlet generating function $U(s)$ of $f(m)$ can be represented as a product

$$
U(s)=\sum_{m=1}^{\infty} \frac{f(m)}{m^{s}}=\zeta(s) g(s) .
$$

On the other hand by the condition of the theorem $f(m)$ is a multiplicative function, which means that its generating function can be represented as Euler product

$$
U(s)=\sum_{m=1}^{\infty} \frac{f(m)}{m^{s}}=\prod_{p}\left(1-\frac{f(p)}{p^{s}}\right)^{-1} .
$$


Comparing the above two expressions of $U(s)$ we conclude that that the Dirichlet series of numbers $a_{m}$ such that $f(m)=\sum_{d \mid m} a_{d}$ is

$g(s)=\sum_{m=0}^{\infty} \frac{a_{m}}{m^{s}}=\frac{1}{\zeta(s)} \prod_{p}\left(1-\frac{f(p)}{p^{s}}\right)^{-1}=\prod_{p} \frac{1-\frac{1}{p^{s}}}{1-\frac{f(p)}{p^{s}}}=\exp \left\{\sum_{p} \sum_{k \geqslant 1} \frac{f\left(p^{k}\right)-1}{k p^{k s}}\right\}$.

Differentiating this expression of $g(s)$ we obtain that this function satisfies differential equation $g^{\prime}(s)=-g(s) \sum_{m=1}^{\infty} \frac{f(m)-1}{m^{s}} \Lambda(m)$. Multiplying both sides of this equation by $\zeta(s)$ and using the fact that the fact that $U(s)=\zeta(s) g(s)$ we obtain an identity

$$
\zeta(s) g^{\prime}(s)=-U(s) \sum_{m=1}^{\infty} \frac{f(m)-1}{m^{s}} \Lambda(m)
$$

or equivalently

$$
\sum_{m=1}^{\infty} \frac{1}{m^{s}} \sum_{k=1}^{\infty} \frac{a_{k} \log k}{k^{s}}=\sum_{k=1}^{\infty} \frac{f(k)}{k^{s}} \sum_{m=1}^{\infty} \frac{f(m)-1}{m^{s}} \Lambda(m) .
$$

Equating the coefficients of $d^{-s}$ in the Dirichlet series on both sides of the above identity and summing over all $d$ such that $d \leqslant m$ we obtain

$$
\begin{aligned}
S(m) & =\sum_{d \leqslant m} \sum_{k \mid d} a_{k} \log k=\sum_{d \leqslant m} \sum_{k \mid d}(f(k)-1) \Lambda(k) f\left(\frac{d}{k}\right) \\
& =\sum_{k \leqslant m}(f(k)-1) \Lambda(k) \sum_{\ell \leqslant m / k} f(\ell)
\end{aligned}
$$

Therefore, recalling that according to the condition of the theorem $|f(m)| \leqslant 1$, and recalling that $f(p)=1$ for $p>n$, we can estimate

$$
\begin{aligned}
|S(m)| & \leqslant \sum_{k \leqslant m}|f(k)-1| \Lambda(k)\left|\sum_{\ell \leqslant m / k} f(\ell)\right|=\sum_{k \leqslant m}|f(k)-1|\left[\frac{m}{k}\right] \Lambda(k) \\
& \ll m \sum_{p \leqslant m} \frac{|f(p)-1|}{p} \log p \ll m(\log m)^{1 / \beta}\left(\sum_{p \leqslant n} \frac{|f(p)-1|^{\alpha}}{p} \log p\right)^{1 / \alpha} \\
& \ll m(\log m)^{1 / \beta}(\log n)^{1 / \alpha} \mu_{n}(\alpha),
\end{aligned}
$$

for $m \geqslant 2$. Here we have applied the Cauchy inequality with parameters $\frac{1}{\alpha}+\frac{1}{\beta}=$ 1. Inserting this estimate of $S(n)$ into the inequality of Theorem 2.5 with $\varepsilon=\frac{1}{\beta}$ we get

$$
\frac{1}{n} \sum_{m=1}^{n} f(m)=g\left(1+\frac{1}{\log n}\right)+O\left(\mu_{n}(\alpha)\right)
$$


An easy calculation yields

$$
g\left(1+\frac{1}{\log n}\right)=\prod_{p \leqslant n} \frac{1-\frac{1}{p^{1+1 / \log n}}}{1-\frac{f(p)}{p^{1+1 / \log n}}}=\prod_{p \leqslant n} \frac{1-\frac{1}{p}}{1-\frac{f(p)}{p}}\left(1+O\left(\mu_{n}(\alpha)\right)\right) .
$$

Hence follows the proof of the theorem.

\section{References}

[1] A. Axer. Beitrag zur Kenntnis der zahlentheoretischen Funktionen $\mu(n)$ und $\lambda(n)$. Prace matematyczno-fizyczne, 21:65-95, 1910.

[2] P. D. T. A. Elliott. Probabilistic number theory. II, volume 240 of Grundlehren der Mathematischen Wissenschaften. Springer-Verlag, Berlin, 1980.

[3] G. Halász. Über die Mittelwerte multiplikativer zahlentheoretischer Funktionen. Acta Math. Acad. Sci. Hungar., 19:365-403, 1968.

[4] G. H. Hardy. Divergent Series. Oxford, at the Clarendon Press, 1949.

[5] A. A. Karatsuba. Basic analytic number theory. Springer-Verlag, Berlin, 1993.

[6] B. V. Levin and A. S. Faŭnleı̆b. Multiplicative functions and probabilistic number theory. Izv. Akad. Nauk SSSR Ser. Mat., 34:1064-1109, 1970.

[7] E. Manstavičius. Sums of multiplicative functions and limit theorems in probabilistic number theory. I. Litovsk. Mat. Sb., 19(2):135-151, 213, 1979.

[8] A. Mačiulis. The mean value of multiplicative functions. Litovsk. Mat. Sb., 28(3):495-506, 1988.

[9] A. G. Postnikov. Introduction to analytic number theory, volume 68 of Translations of Mathematical Monographs. American Mathematical Society, Providence, RI, 1988.

[10] A. Tauber. Ein Satz aus der Theorie der unendlichen Reihen. Monatsh. Math. Phys., 8(1):273-277, 1897.

[11] V. Zacharovas. Cesàro summation and multiplicative functions on a symmetric group. Liet. Mat. Rink., 41(Special Issue):140-148, 2001, arXiv:0811.1082 [math.CA] 